\title{
The Rosetta Mission: Comet and Asteroid Exploration
}

\author{
Rita Schulz \\ ESA Research and Scientific Support Department, ESTEC, \\ Keplerlaan 1, NL-2001 AZ Noordwijk, The Netherlands \\ email: rschulz@rssd.esa.int
}

\begin{abstract}
In March 2004 the European Space Agency launched its Planetary Cornerstone Mission Rosetta to rendezvous with Jupiter-family comet 67P/Churyumov-Gerasimenko. The Rosetta mission represents the next step into the improvement of our understanding of comet nuclei naturally following the four successful comet nucleus fly-by missions carried out in the past. It will however not perform a simple fly-by at its target comet, but combines an Orbiter and a Lander Mission. The Rosetta spacecraft will go in orbit around the comet nucleus when it is still far away from the Sun, and escort the comet for more than a year along its pre- and post-perihelion orbit while monitoring the evolution of the nucleus and the coma as a function of increasing and decreasing solar flux input. Different instrumentations will be used in parallel, from multi-wavelength spectrometry to in-situ measurements of coma and nucleus composition and physical properties. In addition the Rosetta Lander Philae will land on the nucleus surface, before the comet is too active to permit such a landing (i.e. at around $r=3 \mathrm{AU}$ ) and examine the surface and subsurface composition as well as its physical properties. Two fly-bys at main belt asteroids have been scheduled for the Rosetta spacecraft during its journey to the comet. The first fly-by at E-type asteroid (2867) Steins was already successfully executed in September 2008. The second and main fly-by at asteroid (21) Lutetia is scheduled for July 2010.
\end{abstract}

Keywords. comets: general, individual (67P/Churyumov-Gerasimenko), minor planets, asteroids

\section{Introduction}

Cometary matter is believed to constitute a unique repository of information on the sources that contributed to the proto-solar nebula, as well as on the condensation processes that resulted in the formation of planetesimals, which later formed larger planetary bodies. Their high content of frozen volatiles and organics make comets particularly interesting in view to understanding solar system ices. However, it is these characteristics which make comets so difficult to study. Direct evidence on the volatiles in a comet nucleus is particularly difficult to obtain, as remote observations, even during fly-by missions, only cover species in the coma, which have been altered by physico-chemical processes such as sublimation and interactions with solar radiation and the solar wind. The Rosetta mission represents one possible solution to the problem by combining two strategies of characterizing the properties of a comet nucleus (Schulz, 2009). The approach of a rendezvous mission with a probe staying close to the nucleus along a major part of the orbit and performing comprehensive remote-sensing and analytical investigations of material from the nucleus and the coma, guarantees by design minimal perturbations of the comet material as analyses are performed in situ, at low temperatures, and in a microgravity environment. Furthermore, nucleus material will be analysed in-situ by the instruments on board the Philae Lander at a time when the nucleus is still at a low state of activity. 


\section{Mission Overview}

On 2 March 2004 Rosetta started its 10-year journey to rendezvous with Jupiterfamily comet $67 \mathrm{P} /$ Churyumov-Gerasimenko with an Ariane-5 launch from Kourou in French Guiana. To acquire sufficient orbital energy for being able to rendezvous with its target comet and go in orbit around the nucleus the spacecraft has performed four planetary gravity assist manoeuvres (Earth 4 Mar. 2005, Mars 25 Feb. 2007, Earth 13 Nov. 2007, Earth 13 Nov. 2009). After the second and third Earth gravity assist there is a close fly-by of the spacecraft at a main-belt asteroid. The first of these close asteroid encounters took place on 5 September 2008, when Rosetta passed E-type asteroid (2867) Steins at a distance of $802.6 \mathrm{~km}$ (Schulz, 2009b). On 10 July 2010 the spacecraft will fly-by (21) Lutetia, a large asteroid with an estimated diameter of $95 \mathrm{~km}$ which has been classified to be either of C-type or of M-type. After this second and last asteroid fly-by Rosetta will move into the outer solar system to rendezvous with its target comet at $\mathrm{r}=$ 4.5 AU in May 2014 and go into close orbit in September 2014, when the comet is at $\mathrm{r}=$ $3.4 \mathrm{AU}$ and $\Delta=2.8$ AU. Rosetta will start the global observation and mapping of the nucleus during which the spacecraft will fly down to distances of a few kilometres from the surface. The landing of Philae on the surface of the comet nucleus is planned for 10 November 2014 when the comet is about 3 AU away from the Sun. After a five day prime Lander mission, both, the Orbiter and the Lander will enter the routine scientific phase, escorting the comet to perihelion (12 August 2015) and beyond. The nominal mission will end on 30 December 2015.

\section{Spacecraft and Payload}

The Rosetta spacecraft is a 3 -axis stabilised aluminium box with dimensions of $2.8 \times$ $2.1 \times 2.0$ metres. The scientific payload is on the top panel (Payload Support Module) either body mounted or attached to one of the deployable booms, while the subsystems are on the base panel (Bus Support Module). The steerable 2.2-m high-gain antenna and the Lander Philae are attached to two opposite sides of the spacecraft and the solar panel wings extend from the other two sides. In the vicinity of comet $67 \mathrm{P} / \mathrm{Churyumov-}$ Gerasimenko the scientific instruments on the orbiting spacecraft point almost always towards the comet, while the antenna and solar arrays point towards the Earth and the Sun. Each solar panel has a length of $14 \mathrm{~m}$ which results in a total area of $64 \mathrm{~m}^{2}$ and a total span of $32 \mathrm{~m}$ from tip to tip. The total launch mass of Rosetta was estimated to be about $2900 \mathrm{~kg}$ including $1720 \mathrm{~kg}$ of propellant, $165 \mathrm{~kg}$ of scientific payload on the orbiter plus $110 \mathrm{~kg}$ for the lander Philae.

The payload of the Rosetta orbiter consists of 11 instruments (Table 1) which conjointly have unprecedented capabilities to study the volatile and the refractory material in the coma and the composition and physical properties of the comet nucleus. The remote sensing suite of instruments characterizes the nucleus surface in a wide wavelength range from the far-UV $(70 \mathrm{~nm})$ to millimetre $(1.3 \mathrm{~mm})$ with high spatial resolutions. On top of determining the size, shape, rotational state, and detailed surface topography the OSIRIS camera system also characterizes sublimation and erosion processes on the nucleus surface. The infrared imaging spectrometer VIRTIS focuses on the detection and characterization of specific signatures such as typical spectral bands of minerals and molecules, to identify and quantify different constituents of comet material. Together with the MIRO instrument it also studies the thermal evolution of the comet nucleus as a function of solar radiation input. MIRO determines at micro-wavelength the surface and near-surface temperatures as well as the temperature gradient in the nucleus. It also 
Table 1. Rosetta Orbiter Payload

\begin{tabular}{|c|c|c|}
\hline Short Name & Objective & Principal Investigator \\
\hline & Remote Sensing & \\
\hline \multirow[t]{2}{*}{ OSIRIS } & Multi-Colour Imaging & H. U. Keller, MPS \\
\hline & (Narrow and Wide Angle Camera) & Katlenburg-Lindau, Germany \\
\hline \multirow[t]{2}{*}{ ALICE } & UV-Spectroscopy & A. Stern, SRI \\
\hline & $(70 \mathrm{~nm}-205 \mathrm{~nm})$ & Boulder, CO, USA \\
\hline \multirow[t]{2}{*}{ VIRTIS } & VIS and IR Mapping Spectroscopy & A. Coradini \\
\hline & $(0.25 \mu \mathrm{m}-5 \mu \mathrm{m})$ & IAS-CNR, Rome, Italy \\
\hline \multirow[t]{3}{*}{ MIRO } & Microwave Spectroscopy & S. Gulkis, NASA-JPL \\
\hline & $(0.5 \mathrm{~mm}$ and $1.3 \mathrm{~mm})$ & Pasadena, CA, USA \\
\hline & Mass Spectrometres & \\
\hline \multirow[t]{4}{*}{ ROSINA } & Neutral Gas and Ion Mass Spectroscopy & H. Balsiger \\
\hline & DFMS: $12-150 \mathrm{AMU}, \mathrm{M} / \Delta \mathrm{M} \sim 3000$ & Univ. Bern, Switzerland \\
\hline & RTOF: 1-350 AMU, M/ $\Delta \mathrm{M}>500$ & \\
\hline & COPS gas density and velocity & \\
\hline \multirow[t]{3}{*}{ COSIMA } & Dust Mass Spectrometer & M. Hilchenbach, MPS \\
\hline & $(\mathrm{SIMS}, \mathrm{m} / \Delta \mathrm{m} \sim 2000)$ & Katlenburg-Lindau, Germany \\
\hline & Dust Flux and Physical Properties & \\
\hline \multirow[t]{2}{*}{ GIADA } & $\overline{\text { Grain Impact Analyser }}$ & L. Colangeli, Oss. Astro. \\
\hline & Dust Accumulator & Capodimonte, Italy \\
\hline \multirow[t]{3}{*}{ MIDAS } & Grain Morphology, nm resolution & W. Riedler, IWF \\
\hline & (Atomic Force Microscope) & Graz, Austria \\
\hline & Radio Experiments & \\
\hline \multirow[t]{2}{*}{ CONSERT } & $\overline{\text { Radio Sounding }}$ & W. Kofman, CEPHAH \\
\hline & Nucleus Tomography & Grenoble, France \\
\hline \multirow[t]{3}{*}{ RSI } & Radio Science Experiment & M. Pätzold \\
\hline & & Univ. Köln, Germany \\
\hline & Comet Plasma Environment, Solar Wind & teraction \\
\hline \multirow[t]{11}{*}{$\mathrm{RPC}$} & Langmuir Probe (LP) & A. Eriksson, IRF \\
\hline & & Uppsala, Sweden \\
\hline & Ion and Electron Sensor (IES) & J. Burch, SRI \\
\hline & & San Antonio, TX, USA \\
\hline & Flux Gate Magnetometer (MAG) & K.-H. Glassmeier, IGEP \\
\hline & & Braunschweig, Germany \\
\hline & Ion Composition Analyser (ICA) & R. Lundin, IRF \\
\hline & & Kiruna, Sweden \\
\hline & Mutual Impedance Probe (MIP) & $\begin{array}{l}\text { J.G. Trotignon, LPCE/CNRS } \\
\text { Orleans, France }\end{array}$ \\
\hline & Plasma Interface Unit (PIU) & C. Carr, Imperial College \\
\hline & & London, UK \\
\hline SREM & Radiation Environment Monitor & \\
\hline
\end{tabular}

measures outgassing rates and isotopic ratios of certain major volatile species by measuring their molecular transitions. The ultraviolet imaging spectrograph ALICE determines production rates of numerous atoms and gas molecules, studies small dust grains and ions in the coma, and characterizes the surface of the nucleus at UV wavelengths.

Four instruments collect and analyse gas and dust samples from the inner coma focussing on different aspects. The ROSINA instrument measures the global molecular, elemental, and isotopic composition of neutral gas and ions by in-situ mass spectroscopy. The in-depth analysis of the dust coma is shared by three instruments. The Grain Impact Analyser and Dust Accumulator, GIADA explores the dust flux evolution and grain dynamic properties with position and time, while the secondary ion mass spectrometer COSIMA performs the in-situ compositional analysis of the coma dust including chemical 
Table 2. The Payload of Lander Philae.

\begin{tabular}{|c|c|c|}
\hline Short Name & Objective & Principal Investigator \\
\hline & Imaging & \\
\hline \multirow[t]{2}{*}{ ÇIVA } & Panoramic imaging & J.P. Bibring, IAS \\
\hline & $\begin{array}{l}\text { Vis-IR microscopic imaging } \\
\text { of samples }\end{array}$ & Orsay, France \\
\hline \multirow[t]{3}{*}{ ROLIS } & Descent and & S. Mottola, DLR \\
\hline & Down-Looking Camera & Berlin, Germany \\
\hline & Composition & \\
\hline \multirow[t]{2}{*}{ APX } & $\overline{\alpha-\mathrm{X} \text {-ray Spectrometer }}$ & G. Klingelhöfer \\
\hline & Elemental surface composition & Univ. Mainz, Germany \\
\hline \multirow[t]{3}{*}{ COSAC } & Evolved Gas Analyser & F. Goesmann, MPS \\
\hline & Molecular composition and & Katlenburg-Lindau \\
\hline & chirality of samples & Germany \\
\hline \multirow[t]{2}{*}{ Ptolemy } & Evolved Gas Analyser & I. P. Wright \\
\hline & $\begin{array}{l}\text { Isotopic composition } \\
\text { of light elements in sample } \\
\text { Physical Properties }\end{array}$ & Open University, UK \\
\hline \multirow[t]{2}{*}{ MUPUS } & Multi-Purpose Sensor for & T. Spohn, DLR \\
\hline & (Sub-)Surface Science & Berlin, Germany \\
\hline \multirow[t]{4}{*}{ SESAME } & Comet Acoustic Surface Sounding & K.J. Seidensticker, DLR \\
\hline & Experiment (CASSE) & Köln, Germany \\
\hline & Dust Impact Monitor (DIM) & I. Apathy, KFKI, Hungary \\
\hline & Permittivity Probe (PP) & W. Schmidt, FMI, Finland \\
\hline \multirow[t]{2}{*}{ SD2 } & Drilling and sampling and & A. Ercoli-Finzi \\
\hline & Distribution Device & Politechnico, Milano, Italy \\
\hline \multirow[t]{2}{*}{ ROMAP } & Magnetic and Plasma Monitoring & U. Auster, IGEP \\
\hline & & Braunschweig, Germany \\
\hline \multirow[t]{2}{*}{ CONSERT } & Radio Sounding & W. Kofman, CEPHAG \\
\hline & Nucleus Tomography & Grenoble, France \\
\hline
\end{tabular}

characterisation of main organic components, present homologous and functional groups, as well as the mineralogical and petrographical classification of inorganic phases. The dimensions and microstructure of individual dust grains are determined by the MIDAS instrument, the first atomic force microscope on a space mission.

The nucleus mass, bulk density, gravity coefficients, and moments of inertia are determined by radio science techniques (RSI) and the deep interior of the comet nucleus is investigated by radio sounding with the CONSERT instrument, which transmits long wavelengths radio waves through the nucleus. The comet plasma environment and its interaction with the solar wind is monitored in-situ by five sensors, which have been combined into one instrument suite sharing common subsystems and run by the Rosetta Plasma Consortium (RPC). The radiation environment is measured by a Standard Radiation Monitor (SREM).

The Rosetta lander Philae carries another 10 scientific experiments (Table 2) and will perform the first in situ analysis of comet nucleus material. Philae investigates in-situ the composition and physical properties of the comet nucleus by measuring the elemental, molecular, mineralogical, and isotopic composition of surface and subsurface material down to a depth of about $20 \mathrm{~cm}$, and determining the nucleus mechanical, electrical, and thermal properties. Also the nucleus close environment, its large scale structure, interior, and activity will be studied.

Detailed descriptions of the Rosetta payload can be obtained in Schulz et al. (2009). 


\section{Concluding Remarks}

The Rosetta rendezvous mission to comet $67 \mathrm{P} /$ Churyumov-Gerasimenko determines the composition and physical properties of a comet nucleus through in-situ measurements and remote sensing observations. The evolution of the comet along the orbit around the Sun is investigated by monitoring the nucleus and the near-nucleus environment from a pre-perihelion distance of about 3.4 AU (orbit insertion) through perihelion passage at 1.24 AU and back out to about 2 AU post-perihelion. Rosetta studies how a comet nucleus develops its activity at large heliocentric distance and how such a process functions for high solar radiation close to the Sun. The chemical, mineralogical and isotopic composition of both, the comet nucleus (near the surface) and the inner coma is determined allowing identification and quantification of the chemical reaction chains by which the observed coma gas species are produced from the original icy material on the nucleus. The mission will provide information on "how a comet works" and lead us to a better understanding of the early solar nebula and the evolution of our planetary system. The study of the chemical and isotopic composition of the ices and the dust grains in the comet provides information on the processes through which these compounds formed and about pre-biotic chemistry. Rosetta help answering the question what role comets played in the evolution of life on Earth.

\section{References}

Schulz, R. 2009a, Solar System Research, 43/4, 343

Schulz, R. 2009b, Bull. Am. Astron. Soc., 41, 563

Schulz, R., Alexander, C., Boehnhardt, H., Glassmeier, K.-H. 2009, Rosetta ESAs Mission to the Origin of the Solar System(Springer Science+Business Media), ISBN 978038777517-3. 